\title{
Radio to TeV Gamma-ray Emission from SN 1006 and Shock Acceleration around its Rim
}

\author{
Naito, T. ${ }^{1}$, Yoshida, T. ${ }^{2}$, Mori, M. ${ }^{3}$ and Tanimori, T. ${ }^{4}$ \\ ${ }^{1}$ NAOJ, Solar Physics Division, Mitaka, Tokyo 181-8588, Japan \\ ${ }^{1}$ Yamanashi Gakuin University, Koufu, Yamanasi 400-8575, Japan \\ ${ }^{2}$ Ibaraki University, Faculty of Science, Mito, Ibaraki 310, Japan \\ ${ }^{3}$ ICRR, University of Tokyo, Tanashi, Tokyo 188-8502, Japan \\ ${ }^{4}$ Tokyo Institute of Technology, Department of Physics, Meguro-ku, Tokyo 152, Japan
}

Received; accepted

\begin{abstract}
We examine the multi-wave-length spectrum of SN1006 utilizing inverse Compton radiation (IC) and $\pi^{\circ}$ decay process. Our results match well one based on IC process, as discussed in the previous papers. Those fit enable us to determine physical parameters around the shock accurately. The obtained strength of magnetic field is $4 \pm 1 \mu \mathrm{G}$ and the maximum accelerated energy in power-law distribution of the particle is $E_{\max }=51(B / 4 \mu G)^{-0.5} \mathrm{TeV}$. Energy density of the required electrons exceeds that of the magnetic field so that the system may not be in equipartition.
\end{abstract}

Key words: radio, X-rays, gamma rays, supernova remnant (SN1006)

\section{Introduction}

Supernova remnants (SNRs) have been widely believed to be an acceleration site for galactic cosmic rays, produced by diffusive shock acceleration in SNR's blast wave (see review of Blandford and Eichler 1987). Radio to gamma ray photons with non-thermal spectra from SNRs are expected via interaction of such high energy particles with the environment. Actually, non-thermal radio emissions are observed from a number of SNRs, and proposed as synchrotron radiation. Gamma ray photons may be produced by bremsstrahlung, inverse Compton (IC), and $\pi^{0}$ decay from p-p collision processes. If the emission mechanism from SNRs is fixed observationally, the spectra of parent particles are estimated, which has valuable information on acceleration mechanism of the cosmic rays.

Recent observations for SNR SN1006 in X rays by ASCA (Koyama et al. 1995) and ROSAT (Willingale et al. 1996) and $\mathrm{TeV}$ gamma rays by CANGAROO (Tanimori et al. 1998) have revealed that the existence of the non-thermal particles. The emission from radio to X-rays is well interpreted by synchrotron radiation by relativistic electrons and that the flux of gamma-rays is likely to be IC radiation by the same electrons (Mastichiadis and de Jager 1996: Yoshida and Yanagida 1997). Applying this model, Tanimori et al. (1998) has estimated the magnetic field strength around the shock surface of the SN1006 as $B=6.5 \pm 2 \mu G$. However, the previous studys did not include morphologic information of the remnant: the radio spectrum and X-ray (Einstein data) they used are from the whole remnant but $\mathrm{TeV}$ emission from northeast (NE) rim only. In this paper, we use the latest observed spectrum containing morphology and concentrate our attention on the non-thermal emission from NE rim (showed in Fig. 1). The model is reexamined using the revised data. We estimate physical parameters such as, strength of magnetic field, the maximum accelerated energy and energy density of the accelerated electrons.

SNRs are expected to be the gamma-rays sources through $\pi^{0}$ decay induced by collision between accelerated protons and swept-up matter. Although cosmic ray proton is dominant in the galaxy, no SNR shows an obvious feature of $\pi^{0}$ decay gamma-rays. In this paper, we analyze upper limit accepted for SN1006.

\section{Results and Discussion}

Primary momentum spectra of electrons and protons which are accelerated in shocks by first-order Fermi mechanism can be assumed as $d N / d E=N_{0}\left(E / m c^{2}\right)^{-\alpha} \exp \left(-E / E_{\max }\right)$, where $m$ is the particle mass and $c$ is the light speed. We assume that particles are accelerated to power law spectrum up to maximum energy $E_{\max }$

For electrons, assuming that the radio and X-ray non-thermal flux is purely due to synchrotron radiation, $\alpha=2.2$ and $\left(E_{\max } / \mathrm{TeV}\right) \sqrt{(B / \mu B)}=101$ are derived, where $B$ are magnetic field strength of the emission region. 


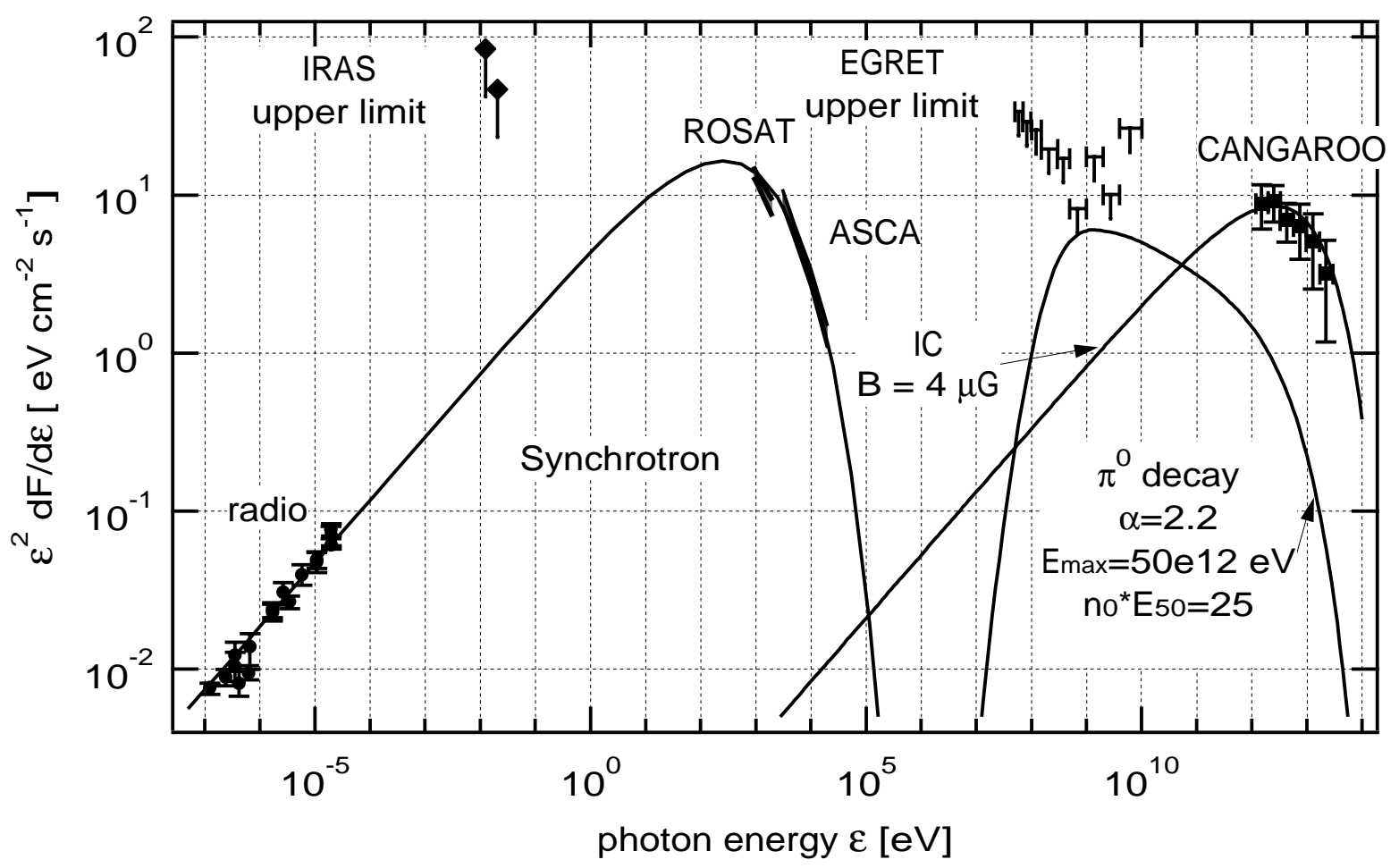

Fig. 1: Multi-band spectrum of energy fluxes observed from NE rim. Radio data is refereed to Reynolds and Ellison (1992) and Winkler and Long (1997). For IR ranges, an IRAS data from Saken et al. (1992) gives upper limit. Willingale et al. (1996) reported that hard flux of ROSAT.dominates in $0.9 \mathrm{eV}-2 \mathrm{keV}$ and is concentrated on NE and SW rims. $A S C A$ observation represents directly the spectrum of NE rim in Koyama et la. (1995). The value of flux is calculated in Ozaki (1998) for energy range from $3 \mathrm{keV}$ to $20 \mathrm{keV}$. Upper limit of gamma rays of the GeV range is calculate from the EGRET archive date (see http://cossc.gsfc.nasa.gov). The spectrum of $\mathrm{TeV}$ gamma rays between $1.5 \mathrm{TeV}$ and 20 $\mathrm{TeV}$ is obtained by observations on the basis of Čerenkov imaging telescope of CANGAROO.

IC spectrum is calculated considering $2.7 \mathrm{~K} \mathrm{CMB}$ and galactic background radiation as target photons. When the synchrotron flux $F_{\text {sync }}$ is fixed, the IC flux $F_{\text {ic }}$ is approximately scaled by parameters as, $F_{\text {ic }} \propto B^{-(\alpha+1) / 2} r$, where $r$ is a ratio of emitting region of $\mathrm{IC}$ to synchrotron. It is odious from Fig. 1 that $B=4 \pm 1 \mu \mathrm{G}$, which corresponds to $E_{\max }=51 \mathrm{TeV}$. This $E_{\max }$ seems a few factors higher than that estimated by standard shock acceleration theory for SN1006 using parameters such as, $u_{\mathrm{s}} \sim 3000 \mathrm{~km} \mathrm{~s}^{-1}, t_{\text {age }} \sim 1000 \mathrm{yr}$. Moreover, the energy density of accelerated electrons surpasses that of magnetic field: the system is not in equipartition in this scenario. These discussions will be appeared in the following full paper.

We also check the contribution of a protons population with the same $\alpha$ and $E_{\max }$ as the electrons, consistent with standard shock acceleration. From upper limit in GeV range, one can see that $\left(E_{\text {tot }} / 10^{50} \mathrm{erg}\right)\left(n_{0} / 1 \mathrm{~cm}^{3}\right) \leq 2.5$, where. $E_{\text {tot }}$ is total energy of accelerated proton $n_{0}$ is mean density of swept-up matter.

Acknowledgements. We thank Dr. M. Ozaki from ASCA group for help in understanding the observational results by $A S C A$. The research related to EGRET observations has made use of data obtained through the High Energy Astrophysics Science Archive Research Center Online Service, provided by the NASA/Goddard Space Flight Center. We thank CANGAROO team for allowing us to use observational results by $C A N G A R O O$ telescope. T. N. is supported by the National Astronomical Observatory as $C O E$ (Center of Excellence) research fellow.

\section{References}

Blandford, R. D., \& Eichler, D.: 1987, Physics Report, 154, 1

Koyama, K., et al.: 1995, Nature, 378, 255

Mastichiadis, A., \& de Jager, O.C.: 1996, Astronomy \& Astrophysics, 311, L5

Ozaki, M.: 1998, ph. D. thesis.

Reynolds, S., P. \& Ellison, D., C.: 1992, Astrophys. J. Lett. , 399, L75

Saken, J., M., Fesen, A., R., \& Shull J., N.: 1992, Astrophys. J. Suppl. , 81, 715

Tanimori, T., et al.: 1998, Astrophys. J. Lett. , 497, L25

Winkler, P., F. \& Long, K., S.: 1997, Astrophys. J. , 491, 829

Willingale, R., et al.: 1996, Mon. Not. R. Astron. Soc. , 278, 749

Yoshida, T., \& Yanagita, S.: 1997, in Proc. 2nd INTEGRAL Workshop 'Transparent Universe', ESA SP-382, 85 